\title{
Guest editors introduction: special issue of the ECMLPKDD 2015 journal track
}

\author{
Concha Bielza $^{1}$ • João Gama ${ }^{2}$ - Alípio Jorge ${ }^{2}$.

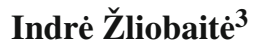

Received: 3 July 2015 / Accepted: 6 July 2015 / Published online: 16 July 2015

(C) The Author(s) 2015

This special issue is a collection of papers submitted to the ECMLPKDD 2015 journal track (and a few delayed from ECMLPKDD 2014) and accepted for publication in Data Mining and Knowledge Discovery.

The European conference on machine learning and principles and practice of knowledge discovery in Databases, ECMLPKDD is a merger of the formerly independent conferences ECML and PKDD.

ECMLPKDD first launched its journal track in 2013. The journal track initiative had large adherence from the community. It was organized again in 2014 and 2015, and will continue in 2016. Two reference journals are involved: Machine Learning and Data Mining and Knowledge Discovery. In addition to being published in the respective journal, papers were presented as regular papers in the conference. Thus, all papers of this special issue were also presented by their authors at the ECMLPKDD 2015 conference in Porto, Portugal, from September 7th to 11th, 2015.

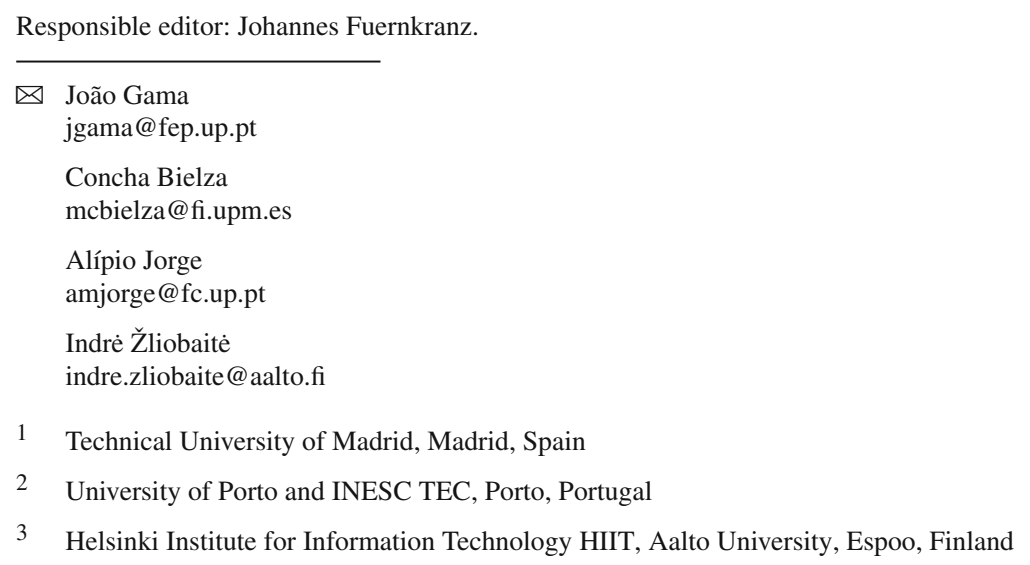


Table 1 Submissions statistics

\begin{tabular}{lr}
\hline Accepted & 10 \\
Rejected & 84 \\
Withdrawn & 1 \\
At authors for revision & 12 \\
Currently under review after revision & 5 \\
Currently under review 1st time & 12 \\
PAPERS RECEIVED (TOTAL) & 124 \\
Papers from 2014 & 4 \\
\hline
\end{tabular}

In the journal track of this conference we look for high-quality submissions that present original and mature work in the areas of data mining and machine learning. However, given the special nature of a conference journal track, only papers that naturally lend themselves to being presented were considered. These requirements exclude, for example, journal versions of previously published conference papers as well as survey papers, which were not considered for the special issue.

The possibility of successive resubmissions of improved versions of a paper has a clear impact on the quality of the accepted papers. This way, the quality of the conference increases to the benefit of participants and readers.

For the 2015 edition, we fostered the continuity of the submission process. The first submission batch started on May 25, 2014, more than one year before the conference. During one year and a half we received batches bi-weekly. Overall, 189 papers were submitted (65 ML + 124 DAMI). As for Data Mining and Knowledge Discovery, at the time of the deadline to send the papers for journal production, ten papers had been accepted. Other 31 papers are still under review. If accepted, these papers will go to the 2016 edition. Moreover, four papers accepted from the 2014 edition are included in this year's special issues. Table 1 summarizes the figures.

The papers included in this special issue of Data Mining and Knowledge Discovery reflect the current hot topics of knowledge discovery. They mostly cover network analysis, clustering, regression and different mining tasks, like episode (set of events) mining, visual data mining and graph mining. The approaches use different tools: partition models, dimensionality reduction, network evolution, directed acyclic graphs, and even tensors. Data to be dealt with cover sequential data, range queries, dynamic and harsh environments, preference data, Internet data and graphs that evolve over time. Finally, applications or potential applications include biological networks and sequences, community search, information cascades, vaccination campaign assessment and brain connectivity analysis.

Figure 1 presents a visual summary, ${ }^{1}$ in the form of a word cloud, generated from the frequent words and bi-grams in the abstracts. Graphs and network analysis seem to be the most relevant words.

This special issue would not have been possible without the help of many people. In particular, we would like to thank the members of the ECMLPKDD 2015 Guest

\footnotetext{
1 Thanks to Carlos Ferreira.
} 


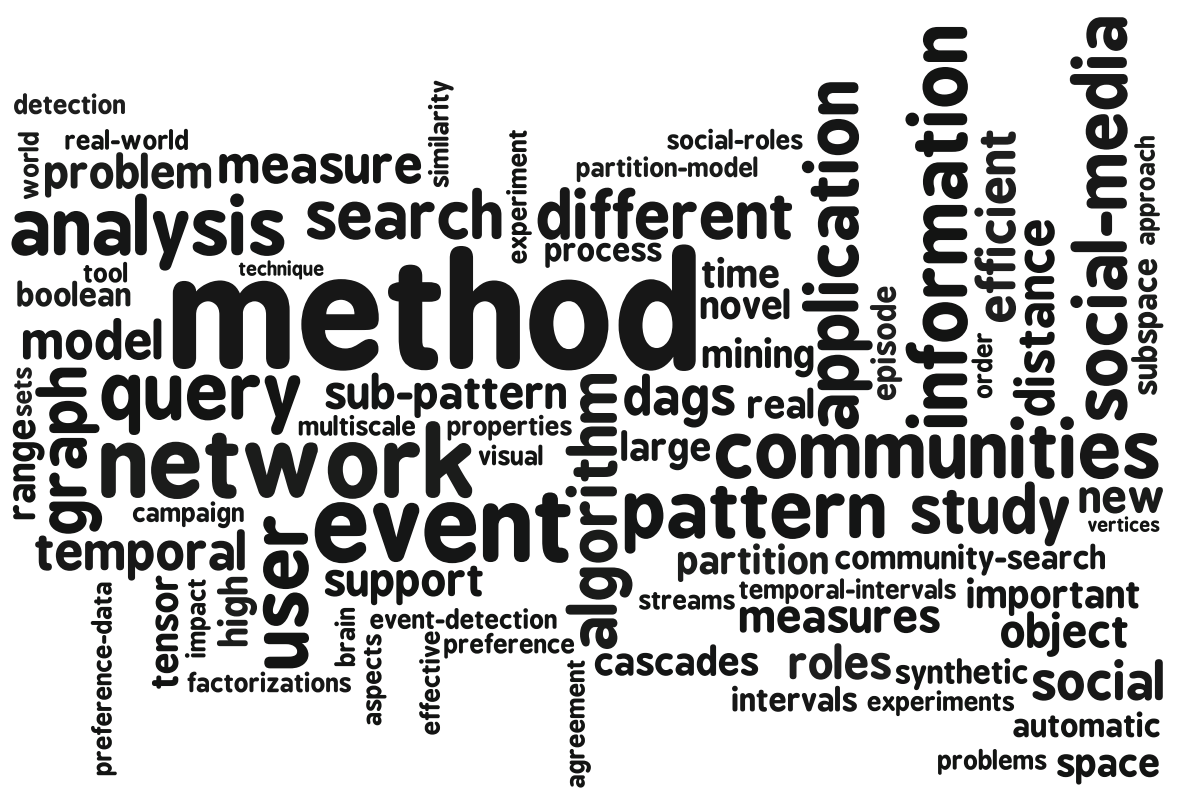

Fig. 1 Word cloud generated from the frequent words and bi-grams in the abstracts

Editorial board, as well as the additional referees for their hard work and timely reviewing of the papers submitted to the special issue. A special thanks to the very active reviewers, to the RAFT team and to Thanh Le Van for the editorial management system. 